\title{
MEMORANDUM
}

RM-4551-NASA

APRIL 1965

\section{NOTE ON THERMAL PROPERTIES OF MARS}

C. Leovy

This research is sponsored by the National Aeronautics and Space Administration under Contract No. NASr-21. This report does not necessarily represent the views of the National Aeronautics and Space Administration. 


\section{PREFACE}

This Memorandum, using available data on Mars, helps to prepare the way for extensive numerical calculations of the general circulation to be conducted later by another RAND researcher. It supports other studies in finding that the Martian surface is finely powdered and demonstrates how radiometric measurements can be used for such purfoses. It was prepared for the National Aeronautics and Space Administration under Contract NASr-21(07). 
$-\mathrm{v}-$

\section{ABSTRACT}

$$
26075
$$

As measured by Sinton and Strong, the variation of infrared emission from the surface of Mars with local time on Mars is here interpreted in terms of a/ simplified theory of diurnal temperature variations, in which the effect of the atmosphere is included. The results suggest a very low thermal conductivity for the upper few centimeters of the Martian ground. Such low conductivities appear to be possible only if the material composing these layers is very fine powder having a characteristic size of not more than a few microns. If a linear relationship is assumed between convective heat transfer and surface temperature, the appropriate constant of proportionality is on the order of $10^{-4} \mathrm{cal}$ per $\mathrm{cm}^{2} \mathrm{sec}$ deg. Afuthow 


\section{CONTENTS}

PREFACE ........................

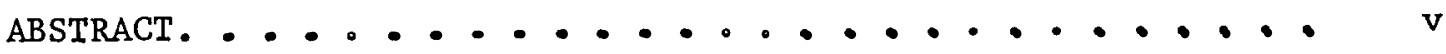

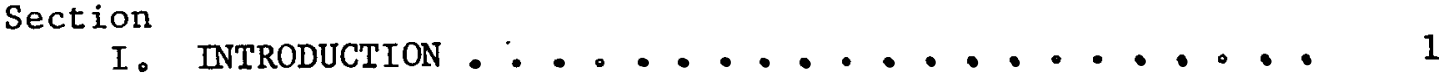

II. DETERMINATION OF THE THERMAL PARAMETERS. . . . . . 2

III. THE MARTIAN GROUND . . . . . . . . . . . . 9

IV. THE MARTIAN ATMOSPHERE . . . . . . . . . . 13

BIBLIOGRAPHY. . . . . . . . . . . . . . 17 


\section{INTRODUCTION}

In analyzing their observations of the variation with local time of the apparent surface temperature on Mars/ Sinton and Strong (1960) find it impossible to match both the amplitude and the phase found in the observations with a theory that neglects conduction and convection of heat into the atmosphere. They suggest that an analysis that includes atmospheric processes could determine something about the meteorology of the planet. This paper is an attempt to carry out such an analysis, using a simple theory of the surface temperature variation, developed by LUnnqvist (1962, 1963). 


\section{DETERMINATION OF THE THERMAL PARAMETERS}

Lonnqvist assumes that a simple heat conduction law with thermal conductivity, $k$, describes the heat flow in the ground, while Newton's law of cooling describes interaction among the surface, atmosphere, and space. Thus the heat balance boundary condition at the ground, $z=0$, is given by

$$
k\left(\frac{d T}{d z}\right)_{z=0}=S-h\left[T_{0}-T_{h}\right],
$$

where $T_{0}$ is the temperature, $S$ is the solar radiation flux, and $h$ and $T_{h}$ are parameters related to radiative processes as well as to conduction and convection in the atmosphere. Their interpretation will be clarified in Sect. 4. Although Eq. (1) is perhaps the simplest boundary condition that can include the effect of atmospheric heat transfer, its applicability to the problem of the terrestrial diurnal temperature wave has been demonstrated by Lonnqvist (1963) under a variety of conditions.

The temperature in the ground and the solar radiation flux are represented by Fourier series in time:

$$
\begin{aligned}
& T=T_{m}+\sum_{j=1}^{\infty}\left(p_{j} \cos j \omega t+q_{j} \sin j \omega t\right) \\
& s=b_{0}+\sum_{j=1}^{\infty}\left(b_{j} \cos j \omega t+a_{j} \sin j \omega t\right),
\end{aligned}
$$


and it is further assumed that $S$ is proportional to the cosine of the angle of incidence of the solar radiation, so that

$$
S=C(\sin \varphi \sin \delta+\cos \varphi \cos \delta \cos \omega t)
$$

for

$$
\tan \varphi \tan \delta+\cos \omega t>0,
$$

$$
S=0
$$

for

$$
\tan \varphi \tan \delta+\cos \omega t \leqq 0,
$$

where $\varphi$ is latitude, $\delta$ is solar declination, $C$ the solar constant, and $w$ the angular frequency of rotation of the planet. Eqs. (4), (4a) are valid if atmospheric attenuation of solar radiation is negligible. From the heat conduction equation, and the boundary condition Eq. (1), it follows that

$$
\begin{gathered}
\left(T_{0}-T_{m}\right)=\frac{n C}{\pi \beta} \sum_{j=1}^{\infty}\left\{j ^ { - 1 / 2 } [ ( 1 + \frac { h } { \beta \sqrt { j } } ) ^ { 2 } + 1 ] ^ { - 1 } \cdot \left[2 j^{-1}(m / n) \sin j \omega t\right.\right. \\
\left.+(j+1)^{-1} \sin (j+1) \omega t+(j-1)^{-1} \cos (j-1) \omega T\right] \cdot[\sin j \omega t \\
\left.\left.+\left(1+\frac{h}{\beta \sqrt{j}}\right) \cos j \omega t\right]\right\}
\end{gathered}
$$


where

$$
\beta=(1 / 2 \rho c k \omega)^{1 / 2},
$$

$\rho$ and $c$ are the density and specific heat of the ground,

$$
\begin{aligned}
& \mathrm{m}=\sin \varphi \cos \delta \\
& \mathrm{n}=\cos \varphi \sin \delta \\
& \tau=\omega^{-1} \cos ^{-1}(-\mathrm{m} / \mathrm{n}),
\end{aligned}
$$

and the mean temperature, $\mathrm{T}_{\mathrm{m}}$, is given by

$$
b_{0}=h\left(T_{m}-T_{h}\right),
$$

in the absence of any diurnally averaged temperature gradient in the ground. It follows that all the properties of the diurnal temperature wave depend on three parameters: $h / \beta, m / n$, and $n c / \pi \beta$. Figure 1 shows the dependence of two of these properties on $h / \beta$ : the phase lag of the maximum temperature after local noon, and the absolute diurnal temperature range in units of $(n C / \pi \beta)$ for the case $m=0$, corresponding to equatorial equinox conditions. We see that both phase lag and temperature range for a given value of $(\mathrm{nC} / \pi \beta)$ decrease monotonically with increasing $h / \beta$; these two curves provide a guide for fitting observed ground temperature variations.

Figure 2 illustrates an attempt to fit the observations of Sinton and Strong by adjusting $(n c / \pi \beta)$ and $(h / \beta)$ with $(m / n)=0$. (Their observations were all within a few latitudinal degrees of the equator.) We see that a good fit to the data is obtained with $(h / \beta)=6$, and $(\mathrm{nC} / \pi \beta)=236.5, \mathrm{~T}_{\mathrm{m}}=226$. A somewhat less satisfactory fit is obtained with $(h / \beta)=10$, and $(n c / \pi \beta)=379$. The figure also shows 


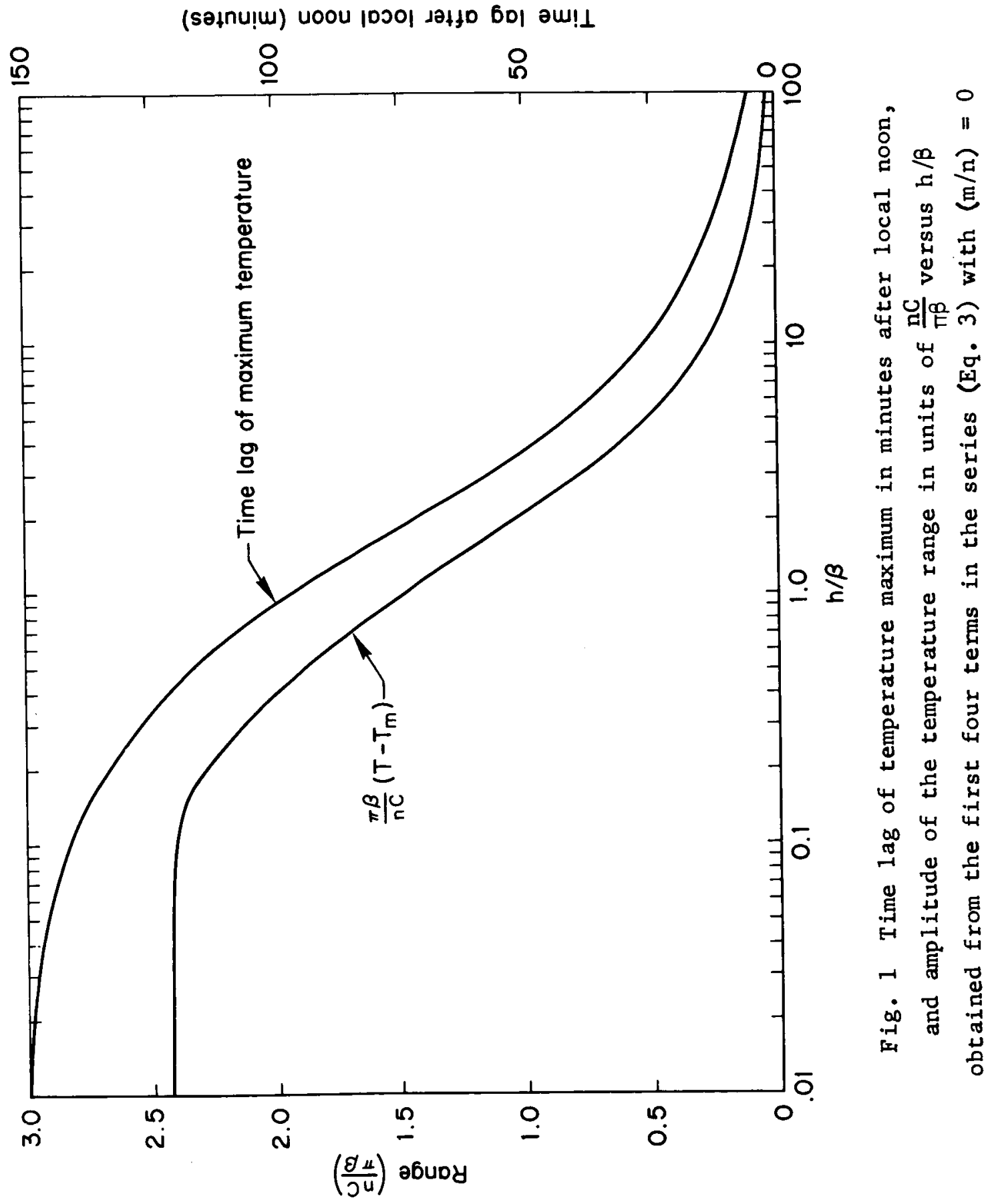




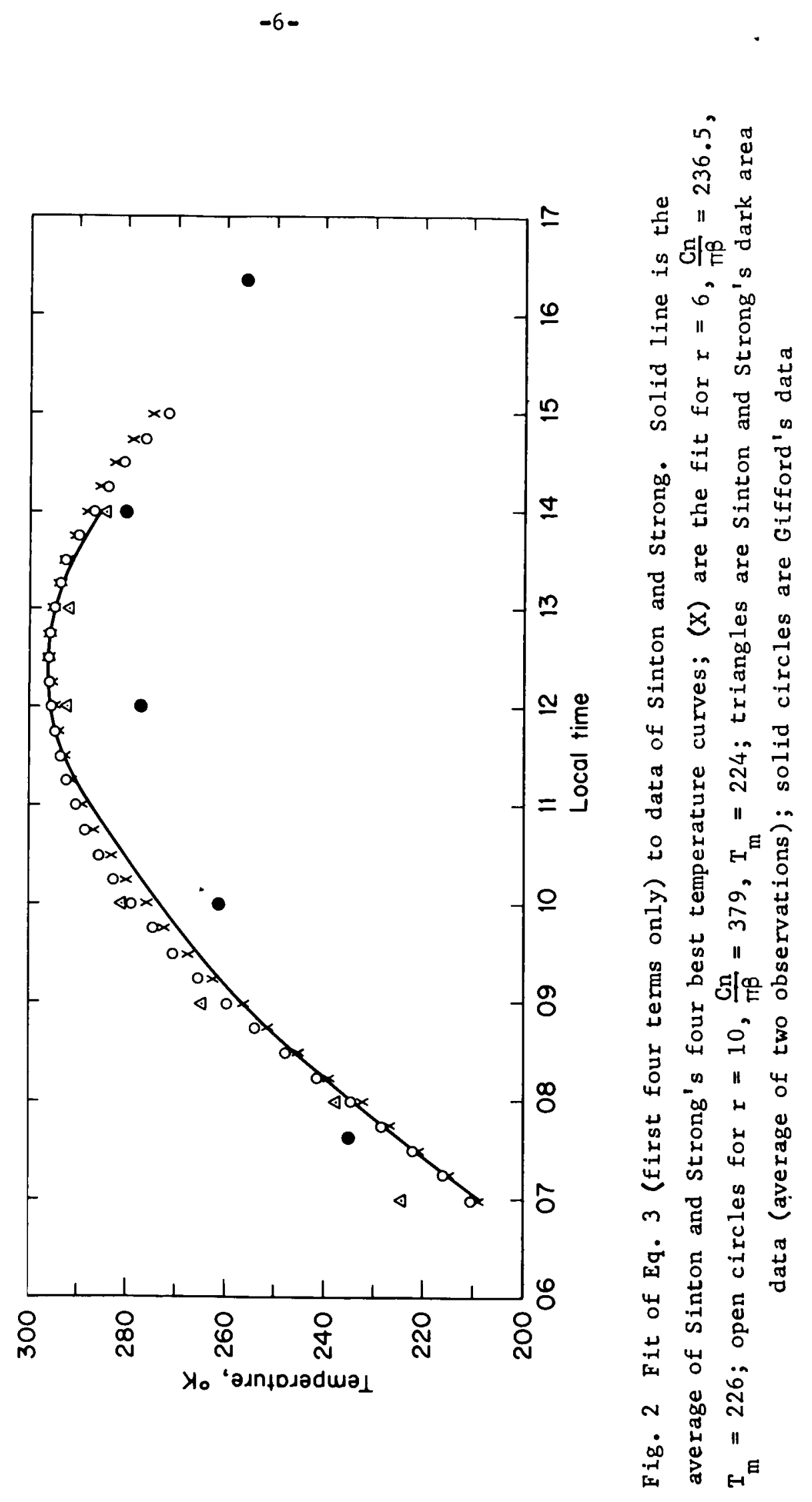


data points from Sinton and Strong for observations mainly on dark areas, and some points from Gifford's analysis (1956) of early Lowe 11 Observatory observations by Coblentz and Lampland. Table 1 summarizes the values of $\mathrm{h},(\rho \mathrm{ck})^{1 / 2}$, and $\mathrm{k}$ if $\rho \mathrm{c}=.30 \mathrm{cals} / \mathrm{cm}^{3}{ }_{\mathrm{K}}$ corresponding to these data; the values for Gifford's data and the dark area data are estimates based on Fig. 1. It has been assumed that the applicable solar constant, corrected for an albedo of .15 , is $0.80 \mathrm{cal} / \mathrm{cm}^{2}$ min. Note that the values of $(\rho c k)^{1 / 2}$ from Sinton and Strong's data lie between the values suggested by these authors when atmospheric effects are neglected $\left[.001<(\rho c k)^{1 / 2}<.004\right]$. These values are therefore not very sensitive to the assumed atmospheric exchange process. 
Table 1

VALUES OF $(h / \beta),(\mathrm{nc} / \pi \beta), \mathrm{h}$, and $(\rho \mathrm{ck})^{1 / 2}$ CORRESPONDING TO FOUR DIFFERENT MODELS OF THE DIURNAL TEMPERATURE

VARIATION OF THE MARTIAN SURFACE

Units $h$ and $(p c k)^{1 / 2}$ are cals cgs

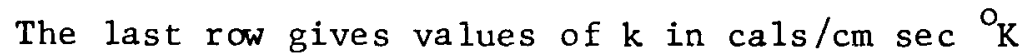

under the assumption that $\rho=2 \mathrm{gms} / \mathrm{cm}^{3}, c=.15 \mathrm{cals} / \mathrm{gm}^{\circ} \mathrm{K}$.

\begin{tabular}{l||c|c|c|c}
\hline Parameter & \multicolumn{2}{|c|}{ Sinton and Strong } & $\begin{array}{c}\text { Sinton and Strong } \\
\text { (dark areas) }\end{array}$ & Gifford \\
\hline $\mathrm{h} / \beta$ & 6 & 10 & 16 & 1.3 \\
\hline $\mathrm{nC} / \pi \beta$ & 236.5 & 379 & 389 & 50 \\
\hline $\mathrm{h}$ & $1.0 \times 10^{-4}$ & $1.0 \times 10^{-4}$ & $1.6 \times 10^{-4}$ & $1.0 \times 10^{-4}$ \\
\hline$(\rho \mathrm{ck})^{1 / 2}$ & .0024 & .0015 & .0015 & .011 \\
\hline $\mathrm{k}$ & $.2 \times 10^{-4}$ & $.8 \times 10^{-5}$ & $.8 \times 10^{-5}$ & $4.2 \times 10^{-4}$ \\
\hline
\end{tabular}


$-9-$

\section{THE MARTIAN GROUND}

The value $\rho c \sim .30 \mathrm{cal} / \mathrm{cm}^{3}{ }^{\circ} \mathrm{K}$ used in deriving the values of $k$ in Table 1 may be considered representative of powdery limonite, or of fine quartz sands. The low values of (pck) ${ }^{1 / 2}$ in Table 1 strongly support the hypothesis that the Martian surface consists of finely divided material. Measurements of polarization (Dolphus, 1961), albedo, and color (Sharonov, 1961) suggest some form of hydrated ferric oxide such as limonite as a primary constituent of the surface, although other materials cannot be ruled out.

Little information is available on the thermal conductivities of dry, finely divided soils. Figure 3, based on Smoluchowski's data as given in International Critical Tables (National Research Council, 1926), shows the pressure variation of the thermal conductivity of six different powders. There is a strong suggestion of two distinct regimes, depending on the pressure: (a) at pressures greater than $50 \mathrm{~mm} \mathrm{Hg}, \mathrm{k}$ has a weak pressure dependence, and its magnitude depends primarily on the particular powder; (b) at pressures less than $30 \mathrm{~mm} \mathrm{Hg}$, $k$ has a strong pressure dependence and depends primarily on particle size, while being almost independent of the particular powder. In the latter regime, over the particle-size range indicated, the approximate relation

$$
\left(k / k_{0}\right)=\left(d / d_{0}\right)^{2 / 3}
$$

(where $\mathrm{d}$ is a characteristic particle dimension) appears to hold. Setting aside Gifford's temperature variation for the moment, the shaded ellipse in Fig. 3 appears to represent for Mars the most 


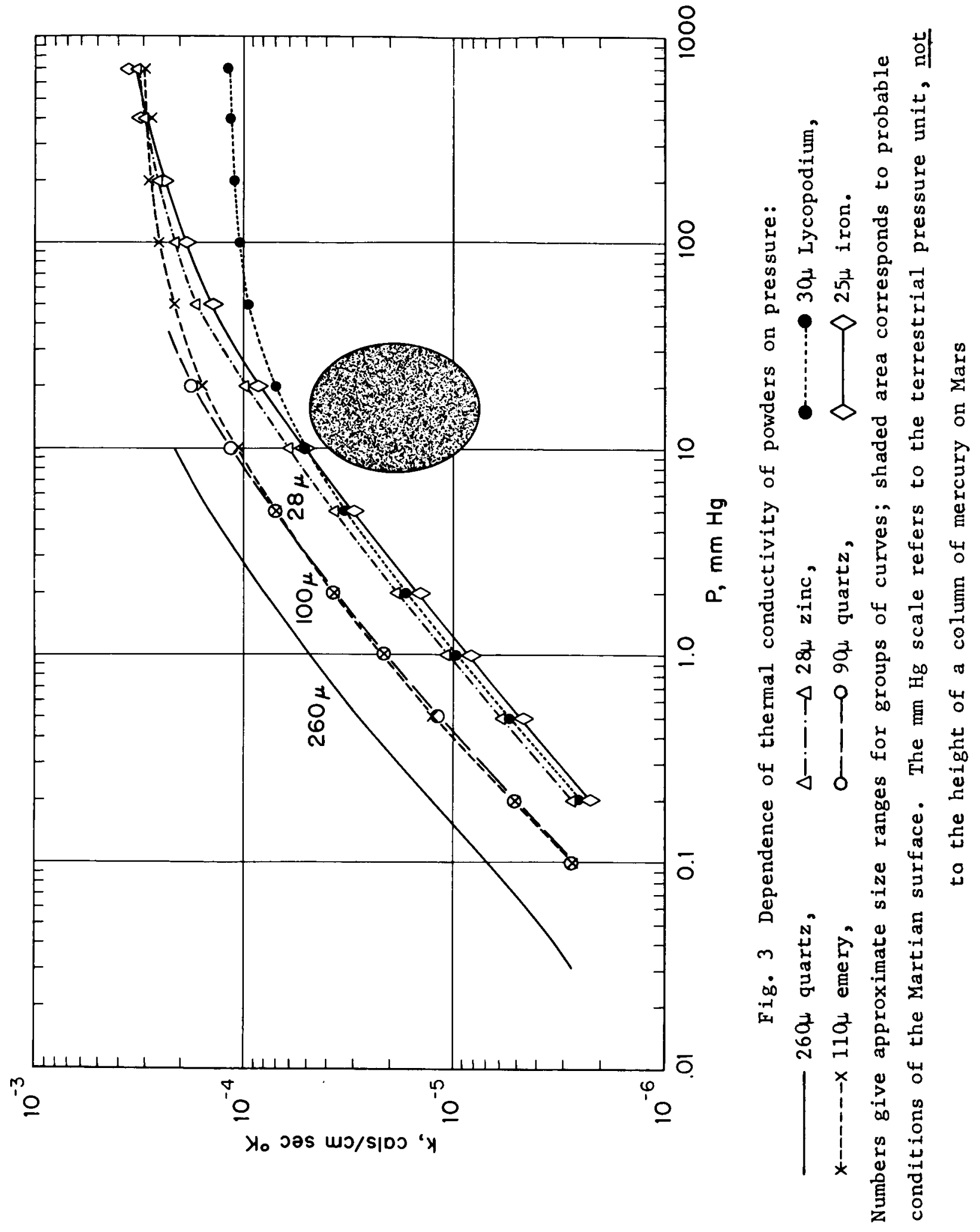


likely range of pressure (Kaplan, Munch and Spinrad, 1964) and thermal conductivity. Assuming that this region is in regime (b) of thermal conductivity, and that Eq. (7) is approximately valid, the deduced thermal conductivity strongly supports the hypothesis that the Martian surface is composed of finely powdered material, predominantly ranging in size from a few tenths of a micron up to about 20 microns. This agrees with polarization measurements (Dolphus, 1961) and estimates of particle-fall velocity for the occasional planetwide yellow cloud obscurations of Mars (Ryan, 1964). The more frequent and less prolonged yellow clouds in the vicinity of the subsolar point require larger particles. According to Ryan (1964), furthermore, it would be increasingly difficult to raise dust clouds as particle size falls below a few hundred microns. It is possible, however, that though the larger particles may exist in relatively limited regions, the small particles predominate and can be raised only under relatively rare conditions of extreme wind.

An even stronger argument exists for assuming that the upper limit on the predominant particle size is in the vicinity of $10 \mathrm{~d}$. For any porosity or grain arrangement, theory and experiment both indicate that the thermal conductivity of porous materials lies between that of the material and that of the substance filling the interstices. For air (as for any combination of nitrogen, $\mathrm{CO}_{2}$, and argon likely to comprise the Martian atmosphere), the thermal conductivity is roughly $5 \times 10^{-5} \mathrm{cals} / \mathrm{cm}{ }^{\circ} \mathrm{K}$ sec. Since this is smaller than $k$ for any known solid, it would give a lower limit to the possible conductivity of the Martian soil if the pores in the 
soil were large enough in comparison with the free path of the interstitial gas molecules that a true continuum conductivity of the gas would apply. The fact that the value of $k$ derived from Sinton and Strong's observations is below this lower limit indicates that the pore size (and hence the grain size) must not be large compared with the free path of the gas molecules -- about $2 \mu$ for $25 \mathrm{mb}$ pressure.

The theory of heat conduction shows that the amplitude of the temperature oscillation is diminished by a factor of ten in a depth of approximately $(4 \mathrm{k} / \rho \mathrm{c} \omega)^{1 / 2}$. Using the values in the first three columns of Table 1 , and the equivalence $\rho c=0.3 \mathrm{ca} 1 / \mathrm{cm}^{3}{ }^{\circ} \mathrm{K}$, we find this depth to be about $3 \mathrm{~cm}$-- the depth for which the deduced $\mathrm{k}$ is representative.

It should be mentioned that Gifford's data suggest particle sizes in the range of several hundred microns. Sinton and Strong's observations are likely to be more representative because they were made with improved techniques, and because Pettit (1961) reports that he was unable to detect a phase lag of the diurnal temperature wave using a technique similar to that used by Coblentz and Lampland in obtaining the data that Gifford discusses. Additional observations to resolve this discrepancy would be useful. 


\section{THE MARTIAN ATMOSPHERE}

Exchange of heat among ground, atmosphere, and space consists of three mechanisms: thermal emission of the ground, back radiation of the atmosphere, and heat transfer by conduction and convection in the atmosphere. Because of the assumption of Newtonian cooling, these heat exchange processes can be written

$$
h\left[T_{0}-T_{h}\right]=\varepsilon \sigma T_{o}^{4}-R_{b}+h_{c}\left[T_{0}-T_{a}\right],
$$

where $\varepsilon$ is the infrared emissivity of the ground, $\sigma$ is Stefan's constant, $R_{b}$ is the flux of back radiation, and $h_{c}$ is a convective heat-transfer coefficient. The parameter $\mathrm{T}_{\mathrm{a}}$ depends on the temperature distribution in the atmosphere. Though $R_{b}$ also depends on the atmospheric temperatures, we assume for the time being that the variation of the relevant atmospheric temperatures is small compared to the variation of ground surface temperature $T_{0}$. Then $R_{b}$ and $T_{a}$ may be taken to be constants. The linear convective heat-flux law can be expected to be a good approximation for forced convection with steady winds (Priestley, 1959, p. 109). Now for temperatures in the range $190--290^{\circ} \mathrm{K}$, the blackbody emission law can be linearized in the form

$$
\epsilon \sigma T^{4}=\epsilon \sigma T^{4}(4.60 x+e)
$$


where $\mathrm{T}=240^{\circ}, \mathrm{x}=(\mathrm{T}-\mathrm{T}) / \mathrm{T}$, and the maximum value of the error, $\mathrm{e}$, is 0.16 . With $\epsilon=0.9$, this leads to

$$
\epsilon \sigma \mathrm{T}_{\mathrm{O}}^{4} \approx-.0117+.000065 \mathrm{~T}
$$

in cal $/ \mathrm{cm}^{2} \mathrm{sec}$. Since $\mathrm{R}_{\mathrm{b}}$ is assumed independent of temperature, this implies $h_{c}=0.35 \times 10^{-4}$ for $h=10^{-4}$, and $h_{c}=0.95 \times 10^{-4}$ for $\mathrm{h}=1.6 \times 10^{-4}$. These are rather small but not unreasonable values. LUnnquist has interpreted his theory in terms of a more detailed analysis of the forced convection regime by Lettau (1951), and he shows that these values of $h_{c}$ would imply a friction velocity of the order of $10 \mathrm{~cm} / \mathrm{sec}$ virtually independent of surface roughness. For very smooth terrain (such as is likely to be found on Mars) under terrestrial conditions, this would imply characteristic winds of a few meters per second at a height of $2 \mathrm{~m}$ (Priestley, 1959, p. 21). The value of $h_{c}$ may be somewhat larger if variations of $\mathrm{T}_{\mathrm{a}}$ and $R_{b}$ with temperature are taken into account. Suppose, for simplicity, that both $\mathrm{T}_{\mathrm{a}}$ and the temperature characterizing back radiation increase linearly with ground temperature $T_{0}$, while the variation of these two temperatures remains smaller than $\mathrm{T}_{0^{\circ}}$ Then

$$
\begin{aligned}
& \mathrm{T}_{\mathrm{a}}=\mathrm{a}+\mathrm{bT} \mathrm{T}_{0}, \\
& \mathrm{R}_{\mathrm{b}}=\mathrm{R}_{\mathrm{bo}}+\mathrm{c}\left(\mathrm{dR}_{\mathrm{b}} / \mathrm{dT}\right) \mathrm{T}_{0},
\end{aligned}
$$

where $a, b, c, R_{b o}$, and $\left(d_{b} / d T\right)$ are constants over the range of Martian temperatures, and $b, c<1$. Assuming that the lower atmosphere is nearly opaque over the main part of the $15-\mu$ bands of $\mathrm{CO}_{2}\left(600--750 \mathrm{~cm}^{-1}\right)$ and is transparent elsewhere, we find that 
$\left(\mathrm{dR}_{\mathrm{b}} / \mathrm{dT}\right) \approx .35 \times 10^{-4} \mathrm{cal} / \mathrm{cm}^{2} \sec { }^{\circ} \mathrm{K}$, and

$$
h_{c} \approx .35 \times 10^{-4}(1+c) /(1-b)
$$

By terrestrial analogy, the representative atmospheric temperatures at heights of a few meters probably do not vary by as much as $50 \%$ of the ground surface temperature, so that these considerations suggest a minimum $h_{c} \sim .35 \times 10^{-4} \mathrm{cal} / \mathrm{cm}^{2} \sec { }^{\circ}$, and a maximum $\mathrm{h}_{\mathrm{c}} \sim 1.1 \times 10^{-4} \mathrm{cal} / \mathrm{cm}^{2} \sec { }^{\circ} \mathrm{K}$.

The forced convection regime probably does not exist throughout the Martian day, especially at times of peak insolation. Nevertheless, these values of $h_{c}$ would give useful limits for parameterizing the gross effects of heat exchange between the Martian ground and atmosphere in studies of the Martian general circulation. 


\section{BIBLIOGRAPHY}

Dolphus, A., "Polarization Studies of Planets," in Planets and Satellites, Vol. 3, G. P. Kuiper and B. M. Middlehurst (eds.), University of Chicago Press, Chicago, 1961, pp. 343-399.

Gifford, F., Jr., "The Surface-Temperature Climate of Mars," Astrophys. J., Vo1. 123, 1956, pp. 154-161.

Kaplan, L. D., G. Münch, and H. Spinrad, "An Analysis of the Spectrum of Mars," Astrophys. J., Vol. 139, 1964, pp. 1-15.

Lettau, H., "Theory of Surface-Temperature and Heat-Transfer Oscillations Near a Level Ground Surface," Trans., Amer. Geophys. Union, Vol. 32, 1951, pp. 189-200.

Lonnquist, 0., "On the Diurnal Variation of Surface Temperature," Tellus, Vol. 14, 1962, pp. 96-101.

-..-, "Further Aspects on the Diurnal Temperature Variation at the Surface of the Earth," Tellus, Vol. 15, 1963, pp. 75-81.

National Research Council, International Critical Tables of Numerica1 Data, Physics, Chemistry, and Technology, Vol. 2, McGraw-Hill Book Company, New York, 1926, P. 315.

Pettit, E., "Planetary Temperature Measurements," in Planets and Satellites, Vol. 3, G. P. Kuiper and B. M. Middlehurst (eds.), University of Chicago Press, Chicago, 1961, pp. 400-428.

Priestley, C. H. B., Turbulent Transfer in the Lower Atmosphere, University of Chicago Press, Chicago, 1959.

Ryan, J. A., "Notes on the Martian Yellow Clouds," J. Geophys. Res., Vo1. 69, 1964, pp. 3759-3770.

Sharonov, V. V., "A Lithological Interpretation of Photometric and Colorimetric Studies of Mars," Soviet Astron., AJ., Vol. 5, 1961, pp. 199-201.

Sinton, W. M., and J. Strong, "Radiometric Observations of Mars," Astrophys. J., Vo1. 131, 1960, pp. 459-469. 